\title{
State-of-the-art overview on biological treatment for CRSwNP anno 2020
}

\author{
Peter Hellings ${ }^{1}$, Wytske Fokkens ${ }^{2}$, and Elisabeth Verhoeven ${ }^{1}$ \\ ${ }^{1}$ University Hospitals Leuven \\ ${ }^{2}$ University of Amsterdam
}

September 10, 2020

\begin{abstract}
Background: The majority of patients with uncontrolled severe CRSwNP, asthma, and atopic dermatitis share a similar T helper 2 type inflammation. This so-called type 2 endotype has given rise to novel treatments targeting specific cytokines driving inflammation in CRSwNP like IL-4, IL-13, IL-5 and IgE. At present, the efficacy of several biological treatments has been demonstrated in CRSwNP Aims: First comprehensive overview of efficacy of reported biologicals for CRSwNP based on published phase 2 and 3 data with focus on the clinically relevant outcome parameters at 16 to 25 weeks of therapy. Methods: After literature search, an overview was made of the reported effects of dupilumab, mepolizumab and omalizumab treatment on patient relevant, i.e. nasal congestion, smell loss and SNOT-22 scores, and patient irrelevant outcome parameters, i.e. CT scan Lund-Mackay, smell test and nasal polyp scores. Therapy duration of 16 to $25 \mathrm{w}$ was chosen for evaluation of efficacy. Results: A direct comparison of efficacy between dupilumab, mepolizumab and omalizumab is challenging given differences in inclusion criteria, outcome parameters and time-points of analyses. However, consistent and major reduction of patient relevant and irrelevant outcome parameters are found in all studies with biologicals for CRSwNP, with most available data on dupilumab. Conclusion: Despite the heterogeneity of protocols, dosages and time-points of analyses of biological trials in CRSwNP, this overview is the first to highlight and present outcomes of biological treatment in a comprehensive way.
\end{abstract}

\section{Hosted file}

FInalversion.PH.2020.09.06.pdf available at https://authorea.com/users/357999/articles/ 480309-state-of-the-art-overview-on-biological-treatment-for-crswnp-anno-2020 